of upper structure above V.o had little effect on H-Wave. Stimulation of Centre median in 2 cases decreased the amplitude of $\mathrm{H}$-Wave.

\title{
4. Electrical Stimulation of the Human Thalamic Nuclei and the Cortical Evoked Responses
}

\author{
Akira Nishimoto, Keizo Matsumoto, Takashi Oнmoto \\ and Shinpei NANBA \\ Department of Neurological School, Okayama University Medical School
}

Electrophysiological studies on evoked cortical activity following stimulatiom of the ventrolateral (VL) and centremedian (CM) nuclei of the thalamus were performed during stereotaxic surgery for 50 parkinsonians and 10 patients with intractable pain.

1) Single stimulation of the VL nucleus of the thalamus demonstrated constantly bilateral activities with first negative (I-N), the second positive-negative (II-PN) and the third negative (III-N) waves which were observed in a series. of sequence from summation of electroencephalographically unseen evoked potentials to clear maximal ones in EEG record.

2) The latency of the I-N wave could be minimized as less as 5-6 msec. bilaterally.

3) As there was proportional correlation between stimulating intensity and amplitude of the I-N wave, it would be supposed to have a rather simple physiological system concerning the pathway from the VL nucleus to the cortex.

4) Peak times were estimated about 8-9 msec. in I-N, 40-50 msec. in the negative phase of the II-PN and $80-100 \mathrm{msec}$. in III-N waves.

5) The II-PN wave of the potential was considered to have close correlation to the VL stimulation. And augmenting response, which was elicited by lowfrequency stimulation of the VL nucleus, was initiated by the development of this: II-PN wave.

6) Although single stimulation of the $\mathrm{CM}$ nucleus was difficult to elicit the evoked potentials, repetitive stimulation elicited recruiting response as similar to that of laboratory animal. 